\title{
Antiproliferative effect of urolithin A, the ellagic acid- derived colonic metabolite, on hepatocellular carcinoma HepG2.2.15 cells by targeting Lin28a/let-7a axis
}

\author{
Zhenpeng Qiu ${ }^{1 *}$, Junxuan Zhou ${ }^{1 *}$, Cong Zhang ${ }^{1}$, Ye Cheng $^{2}$, Junjie $\mathrm{Hu}^{1}$ and Guohua Zheng ${ }^{3}$ \\ ${ }^{1}$ College of Pharmacy, Hubei University of Chinese Medicine, Wuhan, China \\ ${ }^{2}$ Hubei Engineering Research Center of Viral Vector, Wuhan Institute of Bioengineering, Wuhan, China \\ ${ }^{3}$ Key Laboratory of Chinese Medicine Resource and Compound Prescription, Ministry of Education, \\ Hubei University of Chinese Medicine, Wuhan, China
}

\begin{abstract}
An abnormality in the Lin28/let-7a axis is relevant to the progression of hepatitis B virus (HBV)-positive hepatocellular carcinoma (HCC), which could be a novel therapeutic target for this malignant tumor. The present study aimed to investigate the antiproliferative and anti-invasive effects of urolithin A in a stable full-length HBV gene integrated cell line HepG2.2.15 using CCK-8 and transwell assays. The RNA and protein expressions of targets were assessed by quantitative PCR and western blot, respectively. Results revealed that urolithin A induced cytotoxicity in HepG2.2.15 cells, which was accompanied by the cleavage of caspase-3 protein and down-regulation of Bcl-2/Bax ratio. Moreover, urolithin A suppressed the protein expressions of $\mathrm{Sp}-1$, Lin28a, and Zcchc11, and elevated the expression of microRNA let-7a. Importantly, urolithin A also regulated the Lin28a/let-7a axis in transient HBx-transfected HCC HepG2 cells. Furthermore, urolithin A decelerated the HepG2.2.15 cell invasion, which was involved in suppressing the let-7a downstream factors HMGA2 and K-ras. These findings indicated that urolithin A exerted the antiproliferative effect by regulating the Lin28a/let-7a axis and may be a potential supplement for HBV-infected HCC therapy.
\end{abstract}

Key words: Urolithin A; Hepatocellular carcinoma; Lin28a; let-7a; Cell proliferation; HBx

\section{Introduction}

Liver cancer is one of the most malignant tumors globally, with high mortality rates, which commonly result from chronic inflammation and hepatic fibrosis (1). In high-incidence areas of hepatocellular carcinoma (HCC), such as West Africa approximately, $60 \%$ of HCC patients are infected with hepatitis $B$ virus $(\mathrm{HBV})$, suggesting that integrated HBV genetic material in hepatocytes may accelerate the process of cirrhosis and cell immortalization (2). Signaling pathways that activate cell proliferation and invasion are the effectors of $\mathrm{HBx}$, a reverse transcription element in the HBV genome for fibrosis and oncogenesis, interacted by TGF- $\beta$ or other transcriptional factors, such as nuclear factor- $\kappa \mathrm{B}$ and Sp-1 (3-5). Consequently, although HBV replication in infected hepatocytes cannot be eradicated, acting on the $\mathrm{HBx}$-triggered molecular networks and suppressing the cofactors of $\mathrm{HBx}$ might be a potent therapeutic strategy for HBV-infected HCC.

Dysregulated microRNA (miRNA) profiles are the collaborators of $\mathrm{Hbx}$, which aggravate tumor progression involved in carcinogenesis, cell invasion, and metastasis in human malignancies. Let-7a, one of the let-7 family members, was initially characterized in Caenorhabditis elegans with Lin28a, an RNA-binding protein that acted as a suppressor of let-7 expression and controller for development and differentiation (6). Let-7a is also downregulated in $\mathrm{HCC}$ patients and its low expression in liver tissues may contribute to poor survival rates (7). Moreover, Lin28-induced cancer cell EMT is dependent on the low let-7 level and overexpression of the EMT-associated let-7a downstream targets, such as K-ras and HMGA2 (8). Therefore, seeking an approach to alter the Lin28a/let-7a axis in hepatoma cancer cells may lead to the development of effective strategies for HCC therapy.

Urolithins, the dibenzopyran-6-one colonic metabolites derived from ellagic acid (EA) or ellagitannins (ETs), have been suggested to be beneficial for human health. Urolithins in target tissues and cells could act on sub-cellular components and activate signaling transduction. These cell responses

Correspondence: Guohua Zheng: <zgh1227@sina.com>

${ }^{*}$ These authors contributed equally to this work.

Received October 10, 2017| Accepted February 2, 2018 
Table 1. Primers used in real-time PCR.

\begin{tabular}{lcc}
\hline Gene & Forward primer $5^{\prime}$ to $3^{\prime}$ & Reverse primer $5^{\prime}$ to $3^{\prime}$ \\
\hline Lin28a & TTGTCTTCTACCCTGCCCTCT & GAACAAGGGATGGAGGGTTTT \\
B-actin & CCTGGCACCCAGCACAAT & GGGCCGGACTCGTCATACT \\
Let-7a & GGTGAGGTAGTAGGTTGTATAGTT & Uni-miR qPCR primer (TaKaRa) \\
U6 & ACGCAAATTCGTGAAGCGTT & Uni-miR qPCR primer (TaKaRa) \\
\hline
\end{tabular}

contribute to the various biological potentials of EA- and ET-rich diets (9). Based on the anti-proliferative effects of urolithin A on HepG2 cells in our previous findings (10), the function of urolithin A in repressing HepG2.2.15 (HBVintegrated HepG2 cell line) cell proliferation and invasion are discussed in the present study. The results suggested that the regulating effects of urolithin A on the Lin28a/let-7a axis contributed to the inhibition of transcriptional factor Sp-1 and down-regulation of HMGA2 and K-ras in HepG2.2.15 cells.

\section{Material and Methods}

\section{Chemicals}

Urolithin A was synthesized as previously described (11). The purity (>94\%) of urolithin A was evaluated by HPLC, and its molecular weight was confirmed by mass spectrometry analysis (Figure S1). A hydro-soluble tetrazolium salt WST-8 (2-(2-methoxy-4-nitrophenyl)-3-(4-nitrophenyl)-5(2,4-disulfophenyl)-2H-tetrazolium, monosodium salt) was obtained from Dojindo Laboratories (Japan) for CCK-8 assay. All other chemicals and reagents were of analytical grade.

\section{Cell culture}

HepG2 and HepG2.2.15 cells (HepG2 cells integrated with a stable wild-type full-length HBV genome) were obtained from American Type Culture Collection and cultured in Dulbecco's Modified Eagle Medium (Gibco, USA) supplemented with fetal bovine serum (10\%), penicillin $(1 \mathrm{mM})$ and streptomycin $(1 \mathrm{mM})$ at $37^{\circ} \mathrm{C}$ in a humidified $5 \% \mathrm{CO}_{2}$ atmosphere.

\section{Cell viability assay}

The effect of urolithin A on HepG2.2.15 cell viability was evaluated using the CCK-8 assay with WST-8 according to manufacturer's protocol (12). Cell viability values were normalized as follows: [Final absorbance (urolithin A) / Final absorbance (control)] $\times 100 \% . I_{50}$ value for HepG2.2.15 cells was calculated according to a dose-response curve, which was plotted for each concentration.

\section{Quantitative PCR assay for miRNA and mRNA}

Total RNAs containing miRNAs in cells were prepared using mirVana ${ }^{\mathrm{TM}}$ miRNA Isolation Kit (Thermo Fisher Scientific, USA). Real-time quantitative PCR (qPCR) assay was performed on a MiniOpticon ${ }^{\mathrm{TM}}$ (Bio-Rad, USA) system using FastStart Universal SYBR Green Master (Roche, USA).
With initial denaturation at $95^{\circ} \mathrm{C}$ for $120 \mathrm{~s}$, amplifications were performed for 40 cycles at $95^{\circ} \mathrm{C}$ for $5 \mathrm{~s}$ and $55^{\circ} \mathrm{C}$ for 25 s. Primers for qPCR are listed in Table 1.

\section{Western blot}

Western blot was performed as described previously (13). Briefly, separated proteins in SDS-PAGE were transferred onto polyvinylidene fluoride membranes and were sequentially immune-reacted with specific primary antibodies and secondary antibodies. Antibody-conjunct proteins were quantified using SuperSignal ${ }^{\mathrm{TM}}$ West Pico Chemiluminescent Substrate (Thermo Scientific, USA). The primary antibodies cleaved caspase-3 (Cell Signaling Technology (CST, USA)), Bcl-2 (CST), Bax (CST), Lin28a (CST), Sp-1 (CST), HMGA2 (CST), K-ras (CST), Snail (CST), and Zcchc11 (Abcam, UK) were applied after the membranes were blocked in either $5 \%$ milk or $5 \%$ BSA. Anti- $\beta$-actin-peroxidase antibody was obtained from Sigma-Aldrich (USA) and used as an internal reference. The protein content was analyzed using Image Lab 5.1 (Bio-Rad, USA).

\section{Vector and miRNA transfection}

Cells were cultured in 6-well (qPCR and western blot) or 24-well (luciferase assay) plates and were then transfected with plasmids. The Lin28 open reading frame was inserted into the pcDNA3.1 $(+)$ vector (Invitrogen, USA) to express Lin28 (pcDNA3.1-Lin28) with Lipofectamine ${ }^{\mathrm{TM}}$ 3000 (Invitrogen) in HepG2.2.15 cells. Let-7a inhibitor (2'-O-methyl antisense oligonucleotide) and the non-specific control were commercially synthesized as previously described (14). For western blot assay, cells in 6-well plates with approximately $80 \%$ confluency were transfected with $50 \mathrm{nM}$ of let-7a miRNA inhibitor negative control (MOCK) (Thermo Fisher, USA) or $100 \mathrm{nM}$ of inhibitor using Lipofectamine ${ }^{\mathrm{TM}} 3000$ (Invitrogen, USA). Transfection efficiency was validated by $\mathrm{qPCR}$ to directly assess the expression of let-7a.

\section{Transwell invasion assays}

The transwell assay was performed using Matrigelcoated (1:5, BD Biosciences) polycarbonate filters (Corning Costar, USA) (15). Cells were subjected to urolithin A for $24 \mathrm{~h}$ before use, and the chemical was present throughout the assay. Invasive cells in the lower chamber were stained with crystal violet and visualized using an optical microscope. Cell invasion was quantitated via the absorbance 
$(600 \mathrm{~nm})$ of eluted crystal violet with a microtiter plate reader as described previously (16).

\section{Statistics}

All in vitro assessments were performed at least in triplicate. The data are reported as means \pm SD. Differences between experimental groups were analyzed by Student's $t$-test or one-way analysis of variance (ANOVA), followed by Dunnett's $t$-test for multiple comparisons using SPSS (13.0) statistical program. P-values of less than 0.05 were considered statistically significant.

\section{Results}

\section{Urolithin A inhibited cell proliferation and induced cytotoxicity in HepG2.2.15 cells}

In the CCK-8 assay, cells were administrated with urolithin $A$ and $B$ by gradual concentrations (1-120 $\mu \mathrm{M})$ for 24 and $48 \mathrm{~h}$. As shown in Figure 1A, urolithin A repressed cell proliferation in a dose-dependent manner. It was also found that urolithin $A$ induced acute cytotoxicity from
80-120 $\mu \mathrm{M}$, compared to the corresponding controls. However, urolithin B (1-120 $\mu \mathrm{M})$ showed a moderately suppressing effect on HepG2.2.15 cells (Figure S2). Because of this, we chose urolithin $A$ for further investigation. To identify whether urolithin A functions through caspase- 3 signaling, cells were incubated in the absence or presence of Z-DEVD-FMK $(20 \mu \mathrm{M})$ for $6 \mathrm{~h}$ before urolithin $A$ treatment. Data showed that pretreatment with Z-DEVD-FMK abolished the inhibiting effect of cell proliferation induced by urolithin A (Figure 1B). Furthermore, we observed that the protein level of cleaved caspase- 3 was up-regulated and the $\mathrm{Bcl}-2 / \mathrm{Bax}$ ratio was decreased by urolithin $A$ in HepG2.2.15 cells (Figure $1 C$ and $D$ ), suggesting that the suppressing effect of urolithin $A$ on HepG2.2.15 viability could be involved in the activation of caspase-dependent apoptotic signaling.

\section{Urolithin A altered the expressing pattern of Lin28a/ let-7a axis}

Lin28a, which is a repressor of let-7a by recruiting Zcchc11, could be up-regulated by the HBx protein via
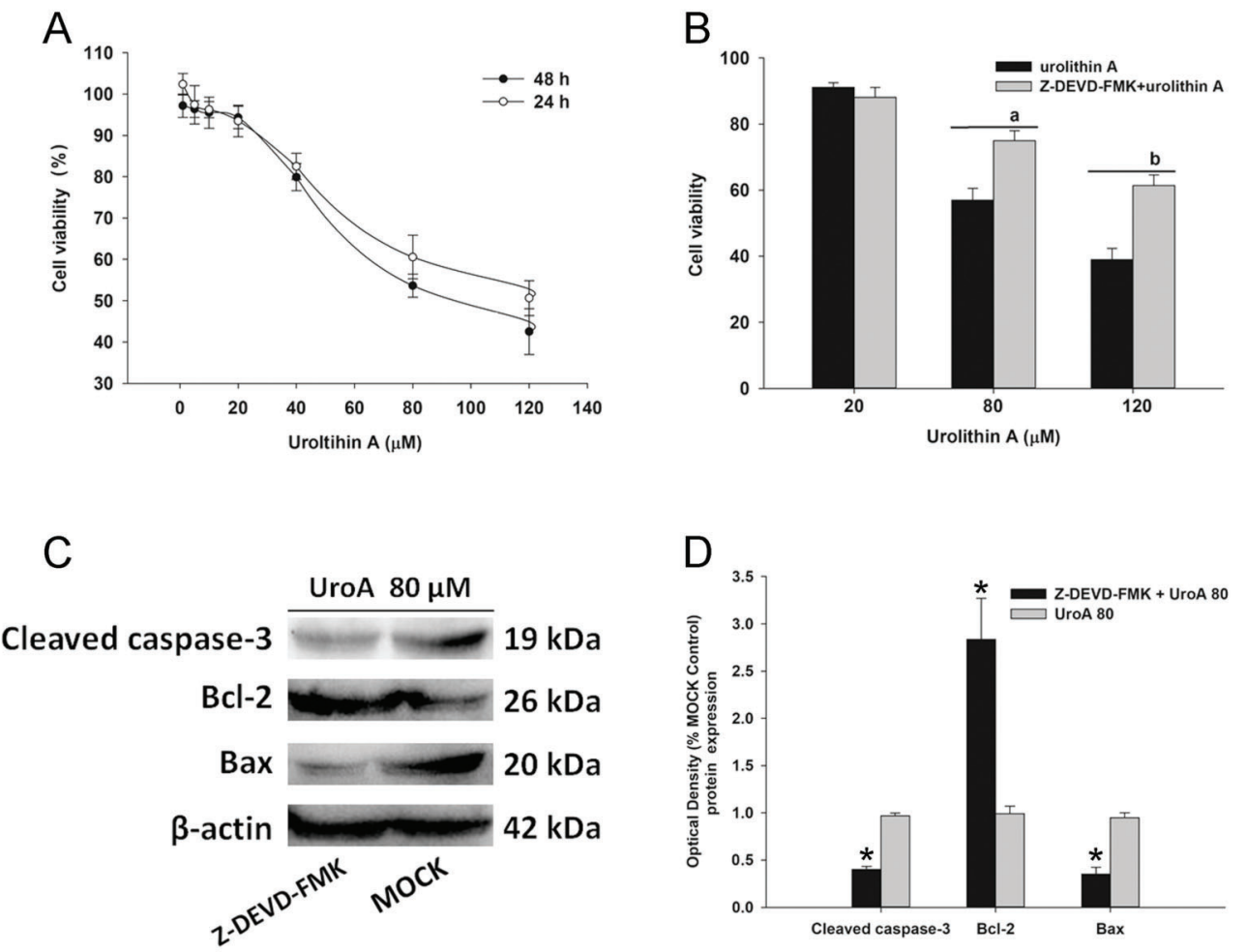

Figure 1. Urolithin A (UroA) suppressed HepG2.2.15 cell proliferation via caspase-3 dependent apoptosis. A, HepG2.2.15 cells were administrated with $0-120 \mu \mathrm{M}$ of urolithin A for 24 or $48 \mathrm{~h}$. Cell viability was assessed by a CCK assay and the data were normalized to normal controls. B, The caspase-3 inhibitor Z-DEVD-FMK alleviated urolithin A-induced HepG2.2.15 cell death (80 or $120 \mu \mathrm{M})$. The cells were incubated in the absence or presence of Z-DEVD-FMK $(20 \mu \mathrm{M})$ for $6 \mathrm{~h}$ prior to urolithin A treatment, and then incubated for $48 \mathrm{~h}$. Data are reported as means $\pm \mathrm{SD}, \mathrm{n}=3$. ${ }^{\mathrm{a}, \mathrm{b}} \mathrm{P}<0.05$ vs 80 or $120 \mu \mathrm{M}$ of urolithin $\mathrm{A}$ group. $C$, and $D$, After urolithin $\mathrm{A}(80 \mu \mathrm{M})$ administration for $48 \mathrm{~h}$, western blot analysis for the protein expressions of cleaved caspase-3, Bcl-2, and Bax was performed in the absence or presence of Z-DEVD-FMK. Data are reported as means $\pm S D, n=3$. ${ }^{*} P<0.05$ compared to the $80 \mu M$ urolithin $A$ group ( $t$-test). 
A
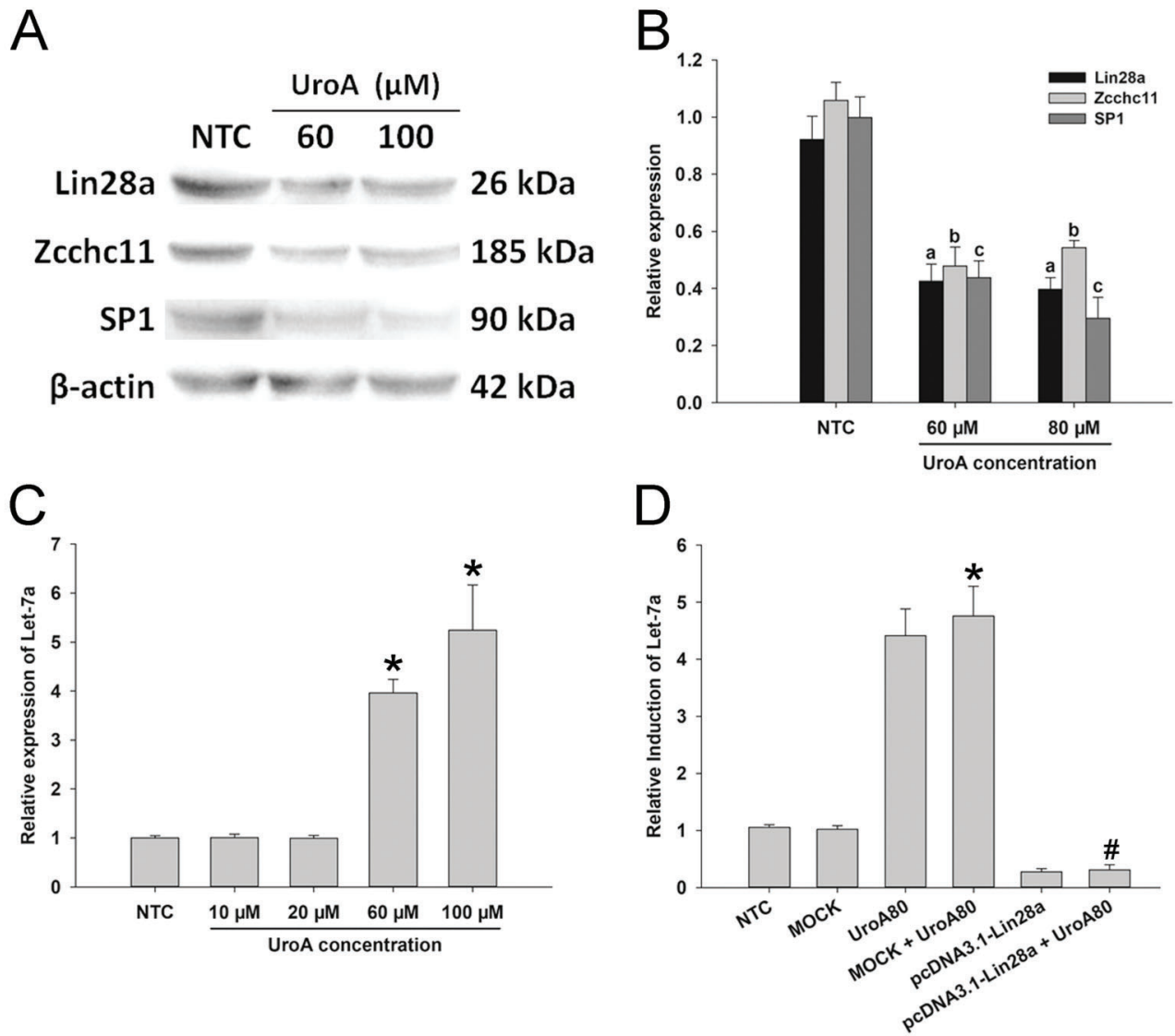

Figure 2. Urolithin A (UroA) regulated the expression of Lin28a/let-7a axis. A, The protein expressions of Sp-1, Lin28a, and Zcchc11 were assessed by western blot assay. $B, \mathrm{Sp}-1$, Lin28a, and Zcchc11 protein levels were semi-quantified by western blot assay. Data are reported as means \pm SD normalized to the corresponding $\beta$-actin values. ${ }^{a, b, c} \mathrm{P}<0.05$ vs the protein expressions of Lin28a, Zcchc11, and Sp-1 in the NTC group, respectively $(n=3)$. C, Cells were treated with urolithin A for $48 \mathrm{~h}$. The let-7a expression was quantitated by qPCR. Data are reported as means $\pm S D(n=3)$. ${ }^{*} P<0.05$ compared to the NTC group. $D$, The overexpression of Lin28a abolished the effect of urolithin A on let-7a expression $(n=3)$. ${ }^{*} P<0.05$ compared to the MOCK control. ${ }^{\#} \mathrm{P}<0.05$ compared to the MOCK + UroA80 group (ANOVA).

Sp-1 in HCC cells. Our data indicated that the protein expressions of Lin28a, Zcchc11, and Sp-1 were suppressed in the urolithin A group, compared to the control group (Figure 2A and $B$ ). We also observed that let-7a was up-regulated when the cells were subjected to urolithin $A$ (Figure $2 \mathrm{C}$ ). To further confirm the effects of urolithin $A$ on the Lin28a/let-7a axis, overexpression of Lin28a (pcDNA3.1Lin28a) by transient transfection was performed in HepG2. 2.15 cells (Figure S3). The results showed that the effects of urolithin $A$ on the expression of let-7a were abolished by Lin28a transfection (Figure 2D), indicating that urolithin A increased the expression of let-7a by targeting Lin28a in HepG2.2.15 cells.

\section{Urolithin A repressed Lin28a expression and elevated the expression of let-7a in transient HBx-transfected HepG2 cells}

The effect of urolithin A on the Lin28a/let-7a axis was also confirmed in transient HBx-overexpressing HepG2 cells. In this section, HepG2 cells $\left(4 \times 10^{5}\right.$ cells/well) were seeded in 6-well plates for $24 \mathrm{~h}$ and transiently transfected with $2 \mu \mathrm{g}$ pcDNA-HBx (pc-HBX) plasmids (pcDNA3.1 (-) plasmid with the sequence encoding for the HBx protein; a gift from Prof. Fan Zhu at Wuhan University). After posttransfection for $24 \mathrm{~h}$, cells were incubated with urolithin $A$ (60 and $100 \mu \mathrm{M})$ for $48 \mathrm{~h}$. As expected, qPCR assay showed that the elevated mRNA expression of Lin28a in the pcDNA-HBx group was repressed by urolithin A (Figure 3A). To examine whether urolithin $A$ affected Lin28a at the protein level in the HBx-transfected HepG2 cells, western blot analysis was performed; the results demonstrated that Lin28a protein expression was suppressed (Figure 3B and $\mathrm{C}$ ). Moreover, the $\mathrm{HBx}$-induced let-7a decrease in the pcDNA-HBx group was alleviated by urolithin $A$ treatment (Figure $3 D$ ). These data indicated that urolithin $\mathrm{A}$ delayed the $\mathrm{HBx}$-induced change of the Lin28a/let-7a axis in HCC cells, further enhancing the findings involved in the effects of urolithin $A$ on Lin28a and let-7a in HepG2.2.15 cells. 


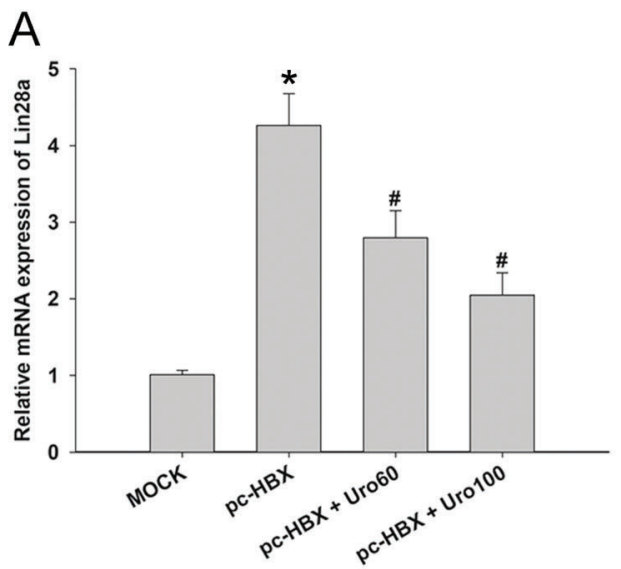

B
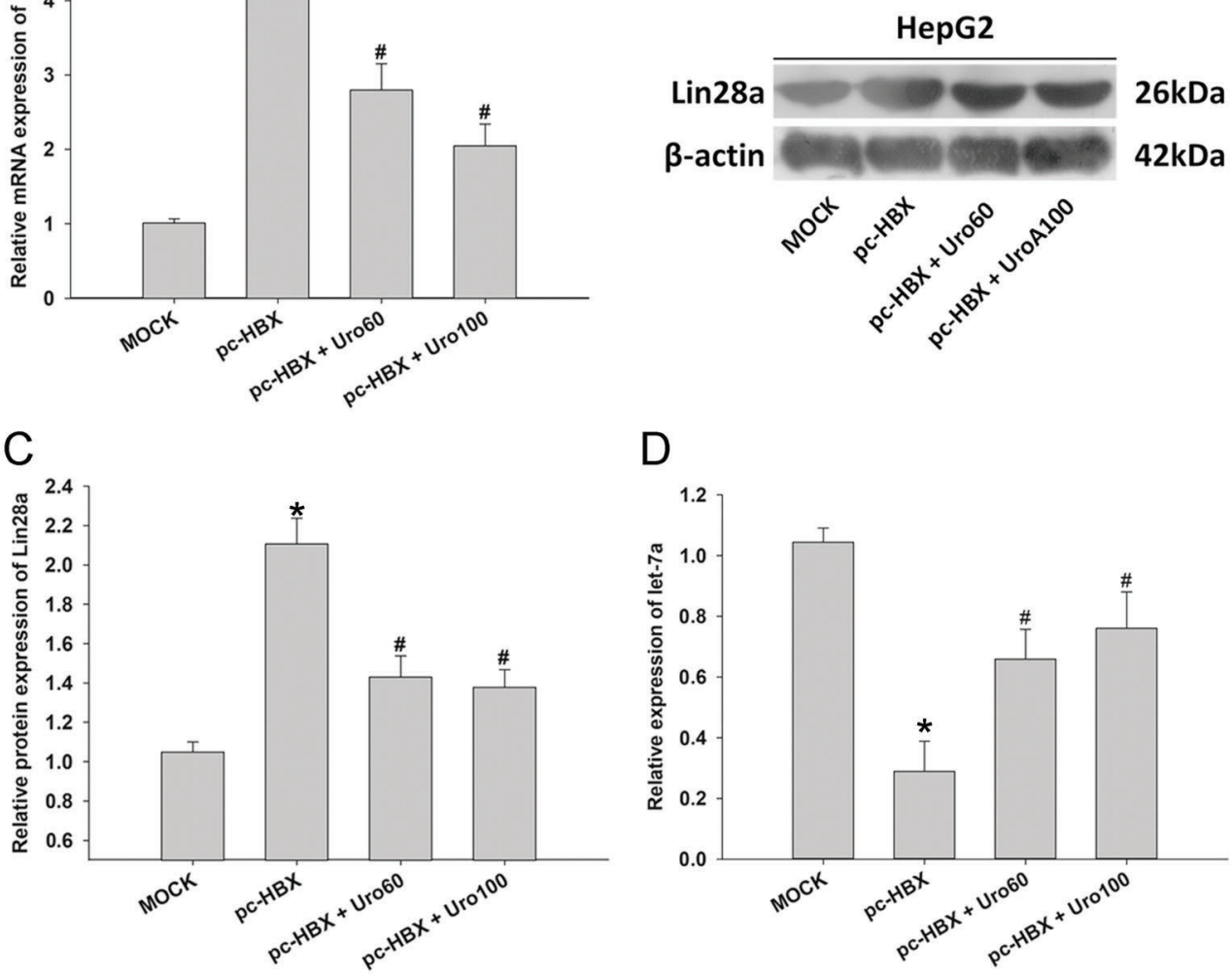

Figure 3. Urolithin A downregulated Lin28a expression and elevated the expression of let-7a in HBX overexpressed hepatocellular carcinoma HepG2 cells. Urolithin A suppressed Lin28a mRNA expression $(A)$ and protein expression $(B$ and $C)$ in HBX-overexpressed HepG2 cells. $D$, Urolithin A elevated the let-7a expression in HBX-overexpressed HepG2 cells (qPCR). ${ }^{*} \mathrm{P}<0.05$ compared to the MOCK control. " $\mathrm{P}<0.05$ compared to the pc-HBX group (ANOVA).

\section{Urolithin A suppressed cell invasion by inhibiting K-ras/HMGA2 signaling in HepG2.2.15 cells}

Since let-7a was increased by urolithin $A$, we assumed that downstream targets of let-7a could respond to urolithin $A$ exposure. Accompanied by an elevation of let-7a (Figure 2C), the expression of HMGA2, a protein enhancing oncogenic transformation and epithelial-mesenchymal transition, was reduced by urolithin $A$ compared to the MOCK group (Figure 4A and B). The effect of urolithin A on cell invasion was evaluated using a transwell assay. As shown in Figure $4 C$ and $D$, cells in the control groups exhibited approximately 3 times higher invasion efficiency than cells in the urolithin A (UroA100) group, indicating that urolithin A could suppress cell invasion in vitro. To further determine whether let-7a elevation contributed to the suppressing effect of urolithin A on cell invasion, the let-7a inhibitor was transfected for $24 \mathrm{~h}$ to abolish the expression of let-7a before urolithin A administration (Figure S4). In Figure 4E, the blockade of let-7a resulted in an increase in HMGA2 protein expression in the western blot assay, compared to the urolithin A group. Moreover, K-ras, which was commonly considered to be a compatible factor of HMGA2 to accelerate cell migration and invasion, was also down-regulated in the urolithin A group (Figure 4A). However, the protein expression of Snail showed no difference between the urolithin $A$ and the MOCK groups. Hence, our data suggested that the regulation of the K-ras/HMGA2 signaling by urolithin $A$ may contribute to the inhibiting effect on cell invasion.

\section{Discussion}

Accepted theory claims that the intestinal metabolism of ETs or EAs to dibenzopyran-6-one derivatives (urolithins) may play an indispensable role in the absorption of ETs. From this perspective, urolithins identified in tissues are important, and their molecular modulation in target cells can be more effective in interpreting the nutritional benefits for human health than that of ETs (17).

Urolithin A is an essential metabolite generated in humans after consumption of EA- and ET-rich food and 

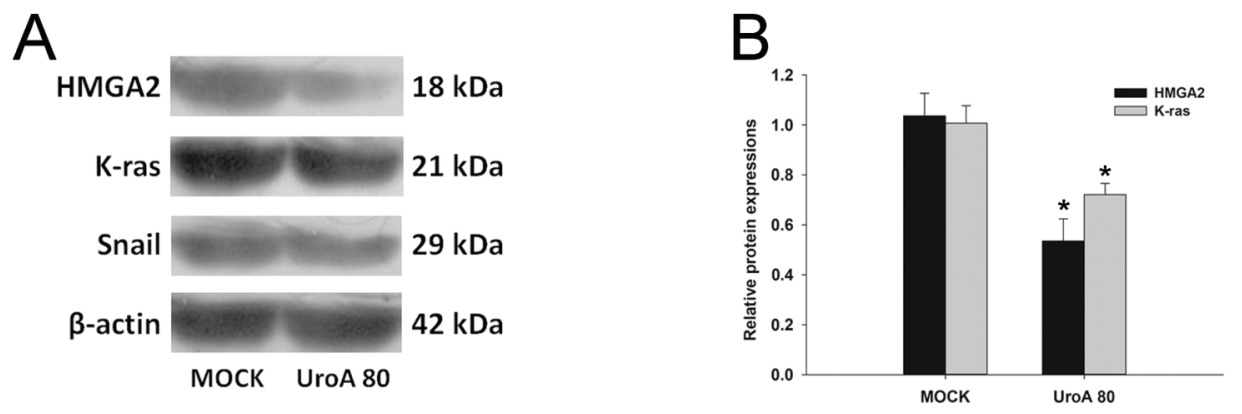

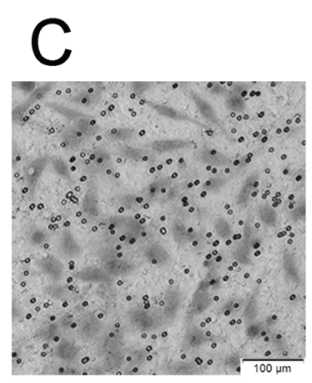

MOCK
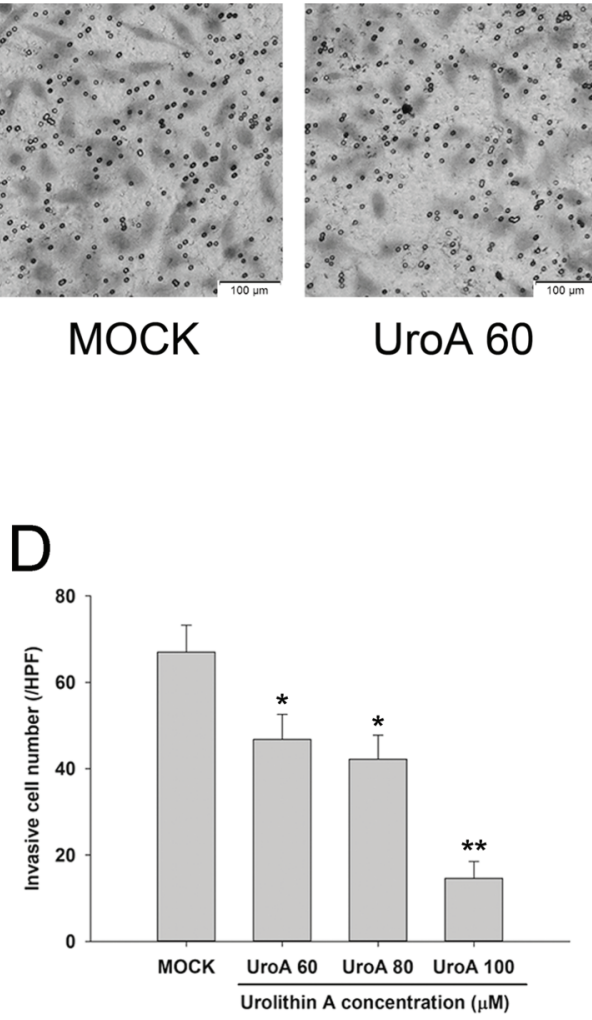

UroA 60

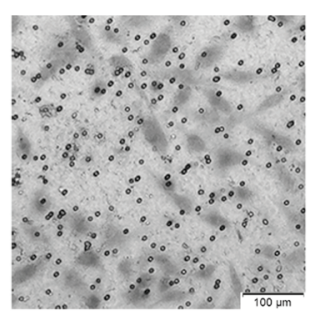

UroA 80

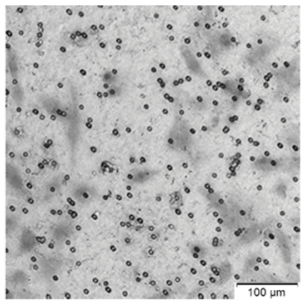

UroA 100

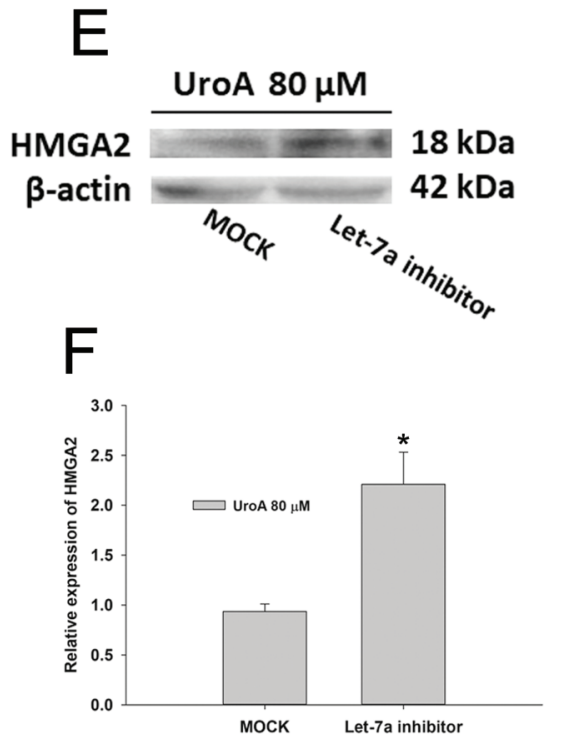

Figure 4. Urolithin $\mathrm{A}$ (UroA) suppressed cell invasion by targeting HMGA2/K-ras axis. $A$, Western blotting analysis of HMGA2, K-ras, and Snail in HepG2.2.15 cells treated by urolithin $A(80 \mu \mathrm{M})$. B, HMGA2 and K-ras protein levels quantified by western blot optical analysis. Quantitative data are reported as means $\pm S D(n=3)$. C , Cell invasion was evaluated by a Matrigel invasion assay after $48 \mathrm{~h}$ incubation with urolithin $\mathrm{A}(60,80$, and $100 \mu \mathrm{M})$. Magnification bar: $100 \mu \mathrm{m}$. The average cell number migrated per high power fields (HPF) is shown in D. Quantitative data are reported as means \pm SD $(n=3)$. $E$, Let-7a inhibitor abolished the suppressing effects of urolithin A on HMGA2 expression by western blot analysis. $F$, Quantitative data from 2 blots shown in $E$. $n=3$. Data are reported as means $\pm S D$. ${ }^{*} \mathrm{P}<0.05,{ }^{* *} \mathrm{P}<0.01$ compared to the MOCK group (ANOVA).

healthy supplements (18). Cell signaling transduction (EGFR, $\beta$-catenin), essential regulators for cell cycle (cyclin D1, c-Myc, p21), and programmed cell death (p53, PARP, caspase-3) could be regulated by urolithins (mainly urolithin A and $B$ ) in cancer cells (19). Though the lipogenic gene in hepatoma Huh7 cells and WNT signaling in HepG2 cells are targets of 
urolithin A or $C(20,21)$, the functions of urolithins in hepatocytes have not been adequately evaluated. Hence, to explore the capacity of urolithin A on HBx-relevant cell invasion, the Lin28a/let-7a axis and Sp-1, a transcriptional factor that could be elevated by $\mathrm{HBx}$, were assumed in the present study to be potential targets of urolithin $A$ in HepG2.2.15 cells.

$\mathrm{Sp}-1$ is an HBx-combinative activator and a member of the Sp/KLF family of transcription factors, and is widely overexpressed in neoplasms, including liver carcinoma $(22,23)$. Based on the anti-proliferative effects of urolithin $A$ in HepG2.2.15 cells, we investigated whether urolithin $A$ could affect the expression of Sp-1. The results revealed that the protein expression level of Sp-1 was repressed by urolithin A treatment. Lin28a, the transcriptional target of $\mathrm{Sp}-1$, which could further elevate the levels of certain cancer-related miRNAs (24), was also suppressed by urolithin A in HepG2.2.15 cells. Therefore, we speculated that suppression of $\mathrm{Sp}-1$ could be responsible for the decrease of Lin28a by urolithin A.

Let-7a functions as a tumor suppressor and is biologically deleted in several cancers, including in HCC patients with HBV infection (25). Moreover, Lin28a suppresses let-7a miRNA biogenesis in tumor cells by recruiting Zcchc11 (TUT4) in the cell cytoplasm to degrading pre-let-7 (26). In our study, urolithin A elevated the let-7a expression, which was delayed by Lin28a overexpression in HepG2.2.15 cells. The similar effects of urolithin A on the Lin28a/let-7a axis were further observed in HBx-overexpressed HepG2 cells. Some evidence has also highlighted that the Zcchc11 downregulation is Lin28b-independent, and should be restricted to Lin28a-positive carcinomas, such as the T47D breast cancer cells (27). In the present study, the protein expression of Zcchc11 was reduced by urolithin A treatment, suggesting that Zcchc11 inhibition plays a bridging role for the effects of urolithin A on the Lin28a/let-7a axis.

High mobility group AT-hook 2 (HMGA2) is a transcription factor highly expressed in the embryonic stage. The exceptional re-expression of HMGA2 by chromosomal rearrangements at chr12q13-15 in neoplasia of mice or

\section{References}

1. Chen X, Calvisi DF. Hydrodynamic transfection for generation of novel mouse models for liver cancer research. $A m \mathrm{~J}$ Pathol 2014; 184: 912-923, doi: 10.1016/j.ajpath.2013.12.002

2. Kirk GD, Lesi OA, Mendy M, Akano AO, Sam O, Goedert JJ, et al. The Gambia liver cancer study: Infection with hepatitis B and $C$ and the risk of hepatocellular carcinoma in West Africa. Hepatology 2004; 39: 211-219, doi: 10.1002/hep.20027

3. Arbuthnot $P$, Kew M. Hepatitis $B$ virus and hepatocellular carcinoma. Int J Exp Pathol 2001; 82: 77-100, doi: 10.1111/ j.1365-2613.2001.iep178.x

4. Tang H, Da L, Mao Y, Li Y, Li D, Xu Z, et al. Hepatitis B virus $X$ protein sensitizes cells to starvation-induced autophagy humans, such as HCC and lung cancer, is involved in the let-7 deletion or loss of let-7-binding sites (28-30). In this study, the western blot analysis supported the notion that urolithin A can down-regulate HMGA2 expression and the capacity is let-7a-dependent. We also found that the invasive potential of HepG2.2.15 cells could be repressed by urolithin $A$ treatment. Let-7a also affected oncogenic K-ras expression by binding the 3'-UTR of K-ras. Exogenous let-7a miRNA in HepG2 cells could specifically reduce abundant K-ras expression (31). The protein expression of K-ras was decreased by urolithin A in HepG2.2.15 cells at the repressible concentration in the transwell assay. Therefore, it is possible that the functions of urolithin $A$ in HepG2.2.15 cells may partly match the above-identified proposal involved in let-7a/HMGA2/K-ras signaling.

In the present study, we described the effects of urolithin $A$ in repressing the proliferation and invasion of the HBV-overexpressed HepG2.2.15 HCC cell line. In fact, the virus replication in HBV-infected patients could not be eliminated, but only alleviated with antiviral agents, such as adefovir. The data in this study could be considered an update of our previous data (10) on HepG2 cells because urolithin A has suppressed cell invasion in HepG2.2.15 cells. In other words, our findings are not only involved in the targets of carcinogenesis but are also based on the initial observation of the $\mathrm{HBx}$-interactional cancerous transcriptional factor, $\mathrm{Sp}-1$. Therefore, our data demonstrated that urolithin A suppressed the HepG2.2.15 cell proliferation and invasion via regulating the Lin28a/let-7a axis and EMTinvolved targets, such as HMGA2 and K-ras.

\section{Supplementary Material}

Click here to view [pdf]

\section{Acknowledgments}

This study was supported by a grant from Research Project of Hubei Provincial Department of Education (Q20162001) to Dr. Zhenpeng Qiu. via up-regulation of beclin 1 expression. Hepatology 2009; 49: 60-71, doi: 10.1002/hep.22581

5. $\mathrm{Ng} \mathrm{SA}$, Lee C. Hepatitis $B$ virus $X$ gene and hepatocarcinogenesis. J Gastroenterol 2011; 46: 974-990, doi: 10.1007/ s00535-011-0415-9

6. Reinhart BJ, Slack FJ, Basson M, Pasquinelli AE, Bettinger JC, Rougvie AE, et al. The 21-nucleotide let-7 RNA regulates developmental timing in Caenorhabditis elegans. Nature 2000; 403: 901-906, doi: 10.1038/35002607

7. Khare S, Zhang Q, Ibdah JA. Epigenetics of hepatocellular carcinoma: role of microRNA. World J Gastroenterol 2013; 19: 5439-5445, doi: 10.3748/wjg.v19.i33.5439 
8. Wang T, Wang G, Hao D, Liu X, Wang D, Ning N, et al. Aberrant regulation of the LIN28A/LIN28B and let-7 loop in human malignant tumors and its effects on the hallmarks of cancer. Mol Cancer 2015; 14: 125, doi: 10.1186/s12943015-0402-5

9. Tomas-Barberan FA, Gonzalez-Sarrias A, Garcia-Villalba R, Nunez-Sanchez MA, Selma MV, Garcia-Conesa MT, et al. Urolithins, the rescue of "old" metabolites to understand a "new" concept: Metabotypes as a nexus among phenolic metabolism, microbiota dysbiosis, and host health status. Mol Nutr Food Res 2017; 61, doi: 10.1002/mnfr.201500901

10. Wang Y, Qiu Z, Zhou B, Liu C, Ruan J, Yan Q, et al. In vitro antiproliferative and antioxidant effects of urolithin $A$, the colonic metabolite of ellagic acid, on hepatocellular carcinomas HepG2 cells. Toxicol In Vitro 2015; 29: 1107-1115, doi: 10.1016/j.tiv.2015.04.008

11. Bialonska D, Kasimsetty SG, Khan SI, Ferreira D. Urolithins, intestinal microbial metabolites of Pomegranate ellagitannins, exhibit potent antioxidant activity in a cell-based assay. J Agric Food Chem 2009; 57: 10181-10186, doi: 10.1021/ jf9025794

12. Ji Q, Hao X, Meng $\mathrm{Y}$, Zhang M, Desano J, Fan D, et al. Restoration of tumor suppressor miR-34 inhibits human p53-mutant gastric cancer tumorspheres. BMC Cancer 2008; 8: 266, doi: 10.1186/1471-2407-8-266

13. Zhou B, Wang J, Zheng G, Qiu Z. Methylated urolithin A, the modified ellagitannin-derived metabolite, suppresses cell viability of DU145 human prostate cancer cells via targeting miR-21. Food Chem Toxicol 2016; 97: 375-384, doi: 10.1016/ j.fct.2016.10.005

14. Khodayari N, Mohammed KA, Goldberg EP, Nasreen N. EphrinA1 inhibits malignant mesothelioma tumor growth via let-7 microRNA-mediated repression of the RAS oncogene. Cancer Gene Ther 2011; 18: 806-816, doi: 10.1038/cgt. 2011.50

15. Zhang L, Tu Y, He W, Peng $Y$, Qu Z. A novel mechanism of hepatocellular carcinoma cell apoptosis induced by lupeol via Brain-Derived Neurotrophic Factor Inhibition and Glycogen Synthase Kinase 3 beta reactivation. Eur J Pharmacol 2015; 762: 55-62, doi: 10.1016/j.ejphar.2015.05.030

16. Petitclerc E, Boutaud A, Prestayko A, Xu J, Sado Y, Ninomiya $Y$, et al. New functions for non-collagenous domains of human collagen type IV. Novel integrin ligands inhibiting angiogenesis and tumor growth in vivo. J Biol Chem 2000; 275: 8051-8061, doi: 10.1074/jbc.275.11.8051

17. Espin JC, Gonzalez-Sarrias A, Tomas-Barberan FA. The gut microbiota: a key factor in the therapeutic effects of (poly) phenols. Biochem Pharmacol 2017, doi: 10.1016/j.bcp.2017. 04.033

18. Cerda B, Tomas-Barberan FA, Espin JC. Metabolism of antioxidant and chemopreventive ellagitannins from strawberries, raspberries, walnuts, and oak-aged wine in humans: identification of biomarkers and individual variability. J Agric Food Chem 2005; 53: 227-235, doi: 10.1021/jf049144d

19. Espin JC, Larrosa M, Garcia-Conesa MT, Tomas-Barberan F. Biological significance of urolithins, the gut microbial ellagic Acid-derived metabolites: the evidence so far. Evid Based Complement Alternat Med 2013; 2013: 270418, doi: $10.1155 / 2013 / 270418$

20. Sharma M, Li L, Celver J, Killian C, Kovoor A, Seeram NP. Effects of fruit ellagitannin extracts, ellagic acid, and their colonic metabolite, urolithin A, on Wnt signaling. J Agric Food Chem 2010; 58: 3965-3969, doi: 10.1021/jf902857v

21. Kang I, Kim Y, Tomas-Barberan FA, Espin JC, Chung S. Urolithin $A, C$, and $D$, but not iso-urolithin $A$ and urolithin $B$, attenuate triglyceride accumulation in human cultures of adipocytes and hepatocytes. Mol Nutr Food Res 2016; 60: 1129-1138, doi: 10.1002/mnfr.201500796

22. Shen Q, Uray IP, Li Y, Krisko TI, Strecker TE, Kim HT, et al. The AP-1 transcription factor regulates breast cancer cell growth via cyclins and E2F factors. Oncogene 2008; 27: 366-377, doi: 10.1038/sj.onc.1210643

23. Safe $S$, Abdelrahim M. Sp transcription factor family and its role in cancer. Eur J Cancer 2005; 41: 2438-2448, doi: 10.1016/j.ejca.2005.08.006

24. You X, Liu F, Zhang T, Lv N, Liu Q, Shan C, et al. Hepatitis B virus $X$ protein upregulates Lin28A/Lin28B through Sp-1/cMyc to enhance the proliferation of hepatoma cells. Oncogene 2014; 33: 449-460, doi: 10.1038/onc.2012.618

25. Li J, Shi W, Gao Y, Yang B, Jing X, Shan S, et al. Analysis of microRNA expression profiles in human hepatitis B virusrelated hepatocellular carcinoma. Clin Lab 2013; 59: 10091015, doi: 10.7754/Clin.Lab.2012.120901

26. Hagan JP, Piskounova E, Gregory RI. Lin28 recruits the TUTase Zcchc11 to inhibit let-7 maturation in mouse embryonic stem cells. Nat Struct Mol Biol 2009; 16: 1021-1025, doi: 10.1038/nsmb.1676

27. Piskounova E, Polytarchou C, Thornton JE, LaPierre RJ, Pothoulakis C, Hagan JP, et al. Lin28A and Lin28B inhibit let-7 microRNA biogenesis by distinct mechanisms. Cell 2011; 147: 1066-1079, doi: 10.1016/j.cell.2011.10.039

28. Mayr C, Hemann MT, Bartel DP. Disrupting the pairing between let-7 and Hmga2 enhances oncogenic transformation. Science 2007; 315: 1576-1579, doi: 10.1126/science. 1137999

29. Lee YS, Dutta A. The tumor suppressor microRNA let-7 represses the HMGA2 oncogene. Genes Dev 2007; 21: 1025-1030, doi: 10.1101/gad.1540407

30. Heo I, Joo C, Cho J, Ha M, Han J, Kim VN. Lin28 mediates the terminal uridylation of let-7 precursor MicroRNA. Mol Cell 2008; 32: 276-284, doi: 10.1016/j.molcel.2008.09.014

31. Tsang WP, Kwok TT. Let-7a microRNA suppresses therapeuticsinduced cancer cell death by targeting caspase-3. Apoptosis 2008; 13: 1215-1222, doi: 10.1007/s10495-008-0256-z 